\title{
La littérature à l'école en Chine
}

Literature at school in China

La literatura en la escuela en China

Wei Chen et Li Qin

\section{CpenEdition}

\section{Journals}

Édition électronique

URL : https://journals.openedition.org/ries/2667

DOI : $10.4000 /$ ries. 2667

ISSN : 2261-4265

\section{Éditeur}

France Education international

Édition imprimée

Date de publication : 1 décembre 2012

Pagination : 47-58

ISSN : 1254-4590

\section{Référence électronique}

Wei Chen et Li Qin, «La littérature à l'école en Chine », Revue internationale d'éducation de Sèvres [En ligne], 61 | décembre 2012, mis en ligne le 06 février 2015, consulté le 05 juillet 2021. URL : http:// journals.openedition.org/ries/2667 ; DOI : https://doi.org/10.4000/ries.2667 


\section{La littérature à l'école en Chine}

\section{Chen Wei Li Qin}

L'histoire de la littérature chinoise peut remonter au $\mathrm{XI}^{\mathrm{e}}$ siècle avant J.-C., à l'époque où sont apparus les premiers poèmes recueillis plus tard dans Classique des vers ${ }^{1}$. Depuis ce jour, notamment depuis la création du système des concours mandarinaux (ou concours impériaux) ${ }^{2}$ en 605 , la littérature a occupé une place primordiale aussi bien dans l'éducation que dans la vie des Chinois. Cette riche et longue tradition littéraire perdure-t-elle encore aujourd'hui dans le contexte de la mondialisation et à la suite d'énormes changements ? La littérature a-t-elle encore une importance particulière à l'école pré-universitaire chinoise ? Quels sont ses objectifs, son statut, son rôle et sa pédagogie? Quelle relation entretient-elle avec d'autres disciplines à l'école? Les réponses que nous essaierons d'apporter seront évidemment incomplètes, tant la Chine est vaste et diverse. Le seul point commun à toutes les régions chinoises en matière d'éducation nationale est que l'école gratuite et obligatoire de neuf ans - cinq années d'école primaire et quatre de secondaire inférieur est aujourd'hui généralisée dans tout le pays, et complétée par trois années de secondaire supérieur. Pour le reste, chaque province a le droit et la responsabilité d'organiser elle-même son enseignement scolaire, sous la direction du ministère de l'Éducation nationale. Pour cette raison, cet article s'appuie notamment sur le cas de la région de Shanghaï, et essaie d'en dégager une idée générale concernant toute la Chine.

\section{L'ENSEIGNEMENT DU YUWEN (语文) À L’ÉCOLE}

En Chine, le «yuwen » est une matière enseignée durant toute la scolarité antérieure à l'université. Mais sa nature et son statut font depuis longtemps l'objet de débats. En effet, le mot "yuwen » se prête lui-même à des interprétations différentes, ce qui explique dans une certaine mesure l'origine du débat en question. Si le premier des deux caractères constituant "yuwen " n'a pas d'ambiguïté ( $y u$ (语) signifie «langue » et, en l'occurrence, la langue chinoise), le second, wen (文), fait référence à au moins quatre notions du reste toutes

1. En chinois : 诗经 Shi Jing. Cette anthologie rassemble 305 poèmes qui vont du XIe au Ve siècles av. J.-C., provenant des royaumes du nord et du sud de la vallée du Fleuve Jaune. On y trouve les plus anciens exemples de la poésie chinoise. 2. En chinois 科举 (kējǔ) : concours visant à sélectionner les fonctionnaires parmi les gens du peuple à l'époque de la Chine impériale. Le premier but de ce concours était de remplacer la transmission aristocratique du pouvoir par une transmission méritocratique. Le système des concours mandarinaux, qui fut institutionnalisé en 605 mais dont l'origine remonte à la dynastie des Han (206 à 220 av. J.-C.), a existé pendant 1300 ans, et fut aboli en 1905 . 
liées à la langue : wenzi (文字, écriture), wenzhang (文章, texte), wenxue (文学, littérature), wenhua (文化, culture). L'enseignement de yuwen en classe concerne-t-il, outre la langue chinoise, le texte, la littérature ou la culture ? Nous abstenant ici de répondre à cette question, nous notons, à travers la combinaison de ces deux caractères, des liens étroits existant entre la littérature d'une part et la langue, le texte et la culture d'autre part. Ainsi nous semble-t-il difficile de nous limiter à la simple étude de la littérature à l'école, puisque ce sujet ne se sépare pas de la langue, et que son enseignement est aussi celui de la culture. Pour cette raison, il nous paraît plus logique de parler tout d'abord non pas de la littérature mais du yuwen à l'école en Chine.

\section{Nature du yuwen}

Si l'enseignement du yuwen en Chine a plus de trois mille ans, le yuwen ne fut, pendant de très longues périodes, pas considéré comme une matière indépendante, car son enseignement se bornait à l'étude de quelques canons philosophiques, littéraires et historiques, et visait pour l'essentiel à préparer les étudiants aux concours impériaux, à l'issue desquels les lauréats auraient la possibilité d'être nommés fonctionnaires. Ce n'est qu'en 1904, avec la promulgation et la mise en application du Programme impérial des études scolaires (le premier programme systématique et complet de l'enseignement de l'histoire de la Chine) par le gouvernement des Qing, que le yuwen acquit son statut de discipline indépendante au plein sens du mot. ${ }^{3}$

Mais ce statut n'a pas permis de trancher le débat sur la nature du yuwen. Certains spécialistes, considérant le yuwen comme un outil de la communication ainsi que de l'apprentissage et du travail, estiment que son enseignement consiste surtout à permettre l'acquisition de connaissances linguistiques et de compétences de compréhension et d'expression, que les textes étudiés ne sont que des exemples - autrement dit, des prétextes au service de cette acquisition. D’autres spécialistes jugent que le yuwen, en tant que véhicule des idées, des sentiments et de la culture, est plutôt caractérisé par son humanité, et que son enseignement doit surtout viser à éveiller et à développer la sensibilité des élèves, à cultiver leur goût esthétique, et à valoriser l'esprit humain. D'autres encore voient dans le yuwen une culture : il faut tout d'abord faire comprendre aux élèves la langue du texte avant de les faire accéder à l'idée et au contenu, puis les amener à prendre de la distance vis-à-vis du texte, afin de parvenir ainsi à une meilleure maîtrise de la langue. En ce sens, l'enseignement de yuwen vise à améliorer la culture linguistique des élèves.

$\mathrm{Au} \mathrm{XXI}^{\mathrm{e}}$ siècle, ce débat sur la nature du yuwen semble aboutir à un consensus, renforcé par la publication de diverses normes par les instances de l'éducation nationale ou régionale. Selon les textes officiels pour l'école primaire

3. Voir GU Zhichuan, 2006. 
et secondaire à Shanghaï, "le yuwen est à la fois un outil important de la communication humaine et une partie constitutive de la culture. Une de ses caractéristiques principales réside dans la cohérence entre son utilité en tant qu'outil et son humanité en tant que culture. ${ }^{4}$

Dans les textes concernant l'enseignement obligatoire, l'enseignement du yuwen à Shanghaï est défini comme "un cours à caractère pratique et à l'intersection de différentes disciplines, visant à l'acquisition et à l'utilisation de la langue chinoise. Dans l'enseignement obligatoire, ce cours a pour mission de faire acquérir aux élèves la compétence d'échanger et de communiquer en langue chinoise, d'absorber et d'assimiler les cultures excellentes, [...] de façon à améliorer leur culture et favoriser leur développement moral. » 5

\section{Les objectifs}

Les textes officiels que nous avons cités montrent qu'aujourd'hui en Chine, le yuwen est désormais considéré comme une discipline humaine où langue, littérature et culture sont interdépendantes et dont aucune des deux dimensions - utilité et humanité - n'est négligée. L'objectif de l'enseignement de yuwen devient par conséquent plus diversifié et plus compliqué. En résumé, il s'agit de :

- faire aimer la langue chinoise, inspirer un enthousiasme durable pour son apprentissage ;

- faire connaître et maîtriser les règles linguistiques ;

- faire acquérir la capacité de lecture autonome des textes en chinois moderne et celle de lecture des textes en chinois classique ${ }^{6}$ à l'aide de notes, de dictionnaires et d'autres documents ;

4. Commission de l'éducation de la municipalité de Shanghai, La norme shanghaïenne du cours de yuwen pour l'école primaire et secondaire (Version d'essai), Éditions de l'éducation de Shanghai, 2004, p. 25.

5. Ministère de l'Éducation nationale de la République populaire de Chine, La norme nationale du cours de yuwen pour l'enseignement obligatoire, Presses de l'Université normale de Beijing, 2011, p. 2.

6. Dans cet article, le terme « classique » est employé dans un sens linguistique et par opposition à « moderne ». Le " chinois classique », qui est une forme traditionnelle de la langue chinoise écrite, fondée sur la grammaire et le vocabulaire d'états anciens de cette langue, couvre deux notions : le gǔwén (古文, chinois archaïque ou ancienne écriture), utilisée du XI ${ }^{\mathrm{e}}$ siècle av. J.-C. au III ${ }^{\mathrm{e}}$ siècle apr. J.-C., et le wényánwén (文言文, chinois littéraire), généralement considéré comme la langue qui a succédé au gǔwén depuis la fin de la dynastie des Han (IIII siècle) jusqu'au début du XXe siècle. Ces deux formes écrites étant restées relativement proches, la distinction entre le gǔwén et le wényánwén est donc floue. Dans le présent travail, nous les désignons sous le seul terme de " chinois classique ». Ce chinois classique, qui a été utilisé jusqu'au début du XXe siècle dans tous les écrits formels en Chine, est remplacé désormais par le chinois moderne ou " chinois écrit standard » (白话文, báihuàwén), qui est essentiellement basé sur le mandarin parlé, et associé au mandarin standard. Depuis le début des années 1920, il est l'écriture la plus usitée en Chine. De nos jours, le chinois classique est parfois utilisé lors de cérémonies ou dans des circonstances formelles. En revanche, certaines œuvres écrites entre le milieu du XIVe siècle et le début du XXe siècle ont été rédigées dans un chinois « vulgaire » basé sur le mandarin parlé et très proche du chinois moderne. La plupart des personnes ayant suivi une scolarité du niveau secondaire sont en principe à même de lire le chinois classique, car cette capacité (de lire, mais non d'écrire) relève de l'enseignement secondaire et des matières faisant l'objet de contrôles et d'examens. Dans les manuels de yuwen, les textes anciens sont accompagnés d'un glossaire explicatif. Les tests consistent généralement en une version d'un texte en chinois classique vers le chinois moderne. De plus, de nombreux travaux littéraires en chinois classique, tels que ceux de poésie Tang, font l'objet de récitation, mémorisation, compréhension et appréciation des élèves selon leur niveau scolaire. Les anciens romans ou nouvelles en chinois vulgaire ne posent généralement pas de difficulté de lecture ni de compréhension à un lycéen. 
- faire acquérir la capacité de communication orale et écrite dans une langue chinoise standard et correcte, selon les circonstances et les besoins;

- faire acquérir une compétence globale d'étude et de réflexion, en proposant des méthodes scientifiques et de bonnes habitudes;

- développer la compétence de raisonnement imagé et logique, éveiller la conscience et l'esprit d'innovation, faire respecter le fait et la vérité ;

- faire connaître la culture et la sagesse de la nation chinoise, apprendre à respecter les cultures des autres nations et à se nourrir de la quintessence de la culture humaine ;

- valoriser le patriotisme, l'esprit national, la bonne moralité et le sens de la responsabilité sociale, faire acquérir une attitude positive et des valeurs correctes sur la vie. ${ }^{7}$

L'enseignement du yuwen vise également à développer la sensibilité et le goût esthétique et se fixe par conséquent une perspective humaniste. Dans ce cadre, les Normes ont défini des sous-objectifs concrets et précis à chaque étape scolaire pré-universitaire, concernant tous les volets évoqués. ${ }^{8}$

\section{Statut du yuwen}

Le yuwen occupe une place très importante dans le programme scolaire chinois et figure, avec les mathématiques et la langue étrangère, sur la liste des trois matières qui absorbent le plus grand nombre d'heures de classe. Par exemple à Shanghai, sur 4862 heures de classe qui s'étalent sur les cinq années de l'école primaire ( $1^{\text {re }}$ à $5^{\mathrm{e}}$ année), le yuwen en occupe 1530 , soit $31,5 \%$ du total, contre respectivement 578 heures $(11,2 \%)$ pour la langue étrangère et les mathématiques. Au collège ( $6^{\mathrm{e}}$ à $9^{\mathrm{e}}$ année) et au lycée ( $10^{\mathrm{e}}$ à $12^{\mathrm{e}}$ année), à cause du nombre croissant des matières à étudier, le poids du yuwen semble diminuer par rapport à l'école primaire (544 heures sur 3604 au collège, soit 17,8 \% du total; et 294 heures sur 2338 au lycée, soit $12,6 \%$ du total), mais il reste toujours sur un pied d'égalité avec les mathématiques ( 575 heures au collège et 294 heures au lycée) et la langue étrangère (544 heures au collège et 294 heures au lycée). ${ }^{9}$

La place primordiale du yuwen se manifeste aussi par son poids dans le concours d'admission à l'université (gaokao). Selon les régions, le concours couvre des matières différentes. Dans la plupart des cas, il concerne cinq matières et totalise un score de 600 points; le yuwen, au même titre que les mathématiques et la langue étrangère, constitue une épreuve incontournable et représente à lui seul 150 points.

7. Voir La norme shanghaïenne... pour l'école primaire et secondaire, op.cit., p. 27-28.

8. Ibid., p. 28-30, et La norme nationale... pour l'enseignement obligatoire, op.cit., p. 7-18.

9. Voir La norme shanghaïenne... pour l'école primaire et secondaire, op.cit., p. 8, p. 10, p. 12. 


\section{LA LITTÉRATURE DANS LE YUWEN}

En Chine, l'accès des élèves à la littérature se fait surtout par l'étude en classe des textes littéraires, aussi bien en chinois classique qu'en chinois moderne, recueillis dans les manuels de yuwen.

\section{Les critères de sélection des textes pour les manuels}

La Norme nationale se contente de fournir une orientation générale tout en prescrivant que "les textes choisis doivent être beaux quant à la forme et au fond, capables de servir de modèles et d'exemples aux élèves, riches en compréhension culturelle et susceptible d'exprimer l'air du temps... ${ }^{10} \mathrm{La}$ Norme shanghaïenne ne dit pas autrement : "La sélection des textes doit tenir compte de leur portée morale, de leur qualité artistique, de leur pertinence scientifique et de leur lisibilité, de façon à présenter aux élèves des œuvres belles quant à la forme et au fond. » De plus, " les textes choisis doivent se caractériser par une langue correcte, une moralité saine, une variété de styles, et peuvent servir de modèles et d'exemples aux élèves. ${ }^{11}$ " Ainsi, les textes littéraires, qui répondent à toutes les exigences prescrites par les textes officiels, deviennent-ils l'une des deux composantes naturelles du yuwen, d'autant plus que la littérature est considérée comme l'intermédiaire le plus approprié pour réaliser l'objectif du cours de yuwen, à savoir la cohérence entre l'utilité et l'humanité.

Mais étant donné que la littérature est souvent considérée comme une notion "implicite», " mouvante » et "floue », et un champ de connaissances "imprécis » et "évolutif», nous avons eu du mal à distinguer, parmi les textes figurant dans les manuels, ceux qui sont "littéraires » de ceux qui ne le sont pas. Faute de critère objectif et unanime, nous préférons, dans la présente étude, nous en tenir aux textes littéraires canoniques retenus par les manuels. Sont donc considérés comme canoniques les textes en "chinois classique " (guwen ou wenyanwen) ou en " chinois moderne " (baihuawen) issus de la plume d'auteurs unanimement reconnus comme étant dignes du titre d' "hommes de lettres ", ainsi que les textes d'écrivains étrangers bénéficiant d'une notoriété littéraire mondiale et traduits en chinois.

À partir de ce critère d'examen, nous avons pu repérer, dans les 24 tomes de manuels de yuwen pour les douze années de scolarité pré-universitaire, 335 textes littéraires canoniques sur un total de 891 leçons ${ }^{12}$. C'est sur cette base que s'effectue l'analyse présente.

10. La norme nationale... pour l'enseignement obligatoire, op.cit., p. 33.

11. La norme shanghaïenne... pour l'école primaire et secondaire, op.cit, 2004, p. 45.

12. Ces chiffres, ainsi que ceux qui figurent dans les tableaux suivants, sont obtenus par les auteurs à partir des dix-huit manuels de yuwen des neuf années de l'école obligatoire et les six manuels des trois années du lycée. Tous ces manuels sont destinés aux écoles de la Région de Shanghaï, et publiés respectivement par la Maison d'édition de l'éducation de Shanghaï (pour l'école primaire et le collège) et les Presses de l'Université normale de la Chine de l'Est (pour le lycée). 


\section{Une première analyse}

Selon les étapes différentes de la scolarité pré-universitaire et en fonction des sous-objectifs de l'enseignement fixés pour chaque étape, la littérature n’a pas la même place dans le yuwen.

Durant les deux premières années de l'école primaire, aucun texte littéraire canonique n'est retenu dans le manuel de yuwen. Ce phénomène s'explique par le fait qu'à cette étape, il s'agit principalement d'apprendre aux écoliers à déchiffrer les caractères chinois et à les prononcer correctement, de façon à ce qu'ils arrivent à lire couramment les textes qui leur sont présentés et à en saisir le sens global. Pourtant, cet accent mis sur l'acquisition de la compétence linguistique des élèves n'exclut pas une "littérarité » relativement haute des manuels, d'autant plus étonnante que ces manuels sont destinés à des enfants âgés seulement de 7 à 8 ans. Cette "littérarité " se manifeste notamment à travers :

- 64 poèmes anciens (surtout de la Dynastie des Tang, du VII ${ }^{\mathrm{e}}$ au $\mathrm{X}^{\mathrm{e}}$ siècle) en chinois classique, qui ne font pas partie des leçons à apprendre et ne sont donc pas l'objet d'une étude approfondie; au lieu de comprendre le sens et l'idée de ces poèmes, les élèves n'ont qu'à savoir les lire et les réciter à haute voix, car ils sont très courts et comportent peu de mots nouveaux, ce qui facilite grandement la reconnaissance des caractères et la mémorisation des mots et des textes par les enfants;

- le fait que la plupart des textes dans les manuels, bien qu'issus d'auteurs anonymes, sont soit adaptés de textes littéraires canoniques, soit écrits sous forme de poème ou d'essai, avec un caractère poétique assez saisissant.

L'étape qui correspond aux trois années suivantes de l'école primaire $\left(3^{\mathrm{e}}\right.$ à $5^{\mathrm{e}}$ année) est considérée comme une transition : la reconnaissance et la prononciation des caractères font progressivement place à l'écriture des mots et à la saisie du sens des textes; les œuvres littéraires canoniques ou leurs extraits, bien qu'encore peu nombreux (42 textes sur un nombre total de 272 leçons), font leur première apparition dans les manuels; les poèmes en chinois classique existent toujours en tant qu'œuvres littéraires supplémentaires à réciter et à mémoriser; les textes qui ne sont pas considérés comme canoniques continuent à faire preuve de la même littérarité que les années précédentes. En outre, les manuels de yuwen commencent à proposer certaines connaissances littéraires de base sur l'histoire de la littérature, les genres littéraires et les expressions toutes faites ayant une forte connotation littéraire.

Dans le secondaire inférieur, la tâche principale du yuwen est de passer progressivement de l'acquisition de la langue à celle de la littérature. Le nombre de textes littéraires canoniques ainsi que leur proportion dans l'ensemble des manuels augmente sensiblement (190 textes sur un nombre de 249 leçons: $76,3 \%$ de textes relèvent de la littérature canonique), notamment pour les textes en chinois classique ( 85 , soit $34,1 \%$ du total des leçons). Parallèlement, 
les poèmes chinois anciens sont désormais inclus dans les leçons des manuels, ce qui suppose que les élèves sont censés non seulement les réciter et les mémoriser, mais encore les étudier attentivement afin d'en saisir le sens et d'en comprendre les idées. Mais l'entrée des poèmes anciens dans les leçons ne supprime pas la présence de ceux qui font partie des œuvres littéraires supplémentaires, ceux que les élèves doivent réciter et mémoriser en dehors des leçons; alors que les textes «non canoniques " qui figurent dans les manuels restent toujours très littéraires, tant sur le plan de la forme que du fond.

$\mathrm{Au}$ secondaire supérieur, les textes littéraires canoniques occupent à peu près la même proportion (74,1\%) dans les manuels de yuwen qu'au collège. Mais d'un côté, la part des travaux de mémorisation diminue (ce que montre la disparition des poèmes anciens supplémentaires des manuels) alors que celle d'analyse et de réflexion augmente, et de l'autre, les textes de théorie et de critique littéraires deviennent plus nombreux. En outre, les manuels insistent sur le lien entre la littérature et d'autres domaines tels que la peinture, la calligraphie, la musique, l'esthétique, la nature, la tradition, voire les sciences et technologies, conformément aux objectifs prescrits. Mais cela entraîne dans une certaine mesure un affaiblissement général de la littérarité de ce cours : on voit en effet apparaître dans les manuels un nombre non négligeable de textes issus de milieux autres que littéraires, certes riches en idées mais moins beaux formellement.

\section{Une analyse plus approfondie}

Les 335 textes littéraires canoniques répartis dans les 24 tomes de manuels de yuwen peuvent être classés en trois grandes catégories : 143 textes en chinois classique (42,7\% du total des textes littéraires canoniques), 132 textes en chinois moderne $(39,4 \%$ du total), et 60 textes d'écrivains étrangers $(17,9 \%$ du total). Pour les deux premières catégories, les trois genres littéraires les plus représentés sont les essais $(55,9 \%$ et $53,0 \%)$, les poèmes $(34,3 \%$ et $18,9 \%)$, les nouvelles et romans $(9,8 \%$ et $12,1 \%)$. Parmi les textes d'écrivains étrangers, les nouvelles et romans représentent $46,7 \%$, les essais $26,7 \%$ et les poèmes $6,7 \%$. Cette différence s'explique, selon nous, par le fait qu'en Chine, les nouvelles et romans étrangers sont le genre le plus traduit et donc le mieux accepté.

Ces textes de littérature étrangère vont de l'Antiquité à nos jours et sont issus de 50 écrivains provenant de 18 pays et régions du monde. Un point mérite notre attention: Youjin, écrivain singapourienne contemporaine qui a deux textes retenus dans les manuels, est non seulement la seule auteure vivante parmi les huit écrivains les plus choisis, mais aussi la seule, parmi les 50 écrivains étrangers retenus, à écrire en chinois. Son cas, bien qu'unique, reflète l'ouverture croissante du cours de yuwen au monde actuel. 


\section{La littérature et le gaokao}

À l'heure actuelle, la littérarité constitue le caractère le plus évident du test de yuwen au concours d'admission à l'université chinoise (gaokao). Dans celui de la région shanghaïenne de juin 2012, le test de yuwen était composé de deux grandes parties : la lecture ( 80 points) et la composition (70 points). Sur les 26 questions de la partie "lecture ", vingt touchaient la littérature et représentaient un score de 64 points. Quant à la composition, elle exige implicitement des candidats qu'ils mettent en ouvre des procédés de création littéraire.

Dans l'ensemble, le caractère littéraire du test de yuwen au gaokao se manifeste dans les aspects suivants :

- la littérature fusionne de plus en plus avec les connaissances linguistiques : dans la partie du test liée à la compétence linguistique, une quantité croissante de matériau utilisé dans les questions est tirée des textes littéraires canoniques ;

- les connaissances littéraires font l'objet de questions séparées et couvrent tous les genres littéraires, toutes les périodes historiques ainsi que les auteurs de toutes les nationalités ;

- la partie du test liée à la lecture concerne des œuvres littéraires en chinois classique et en chinois moderne, et requiert non seulement la compétence de compréhension et d'analyse mais aussi, et de plus en plus, celle d'appréciation.

Le gaokao étant en Chine un bon instrument de mesure indiquant la tendance de l'évolution de l'enseignement et de l'apprentissage, la place qu'occupe la littérature dans le test de yuwen rehausse indéniablement son statut à l'école chinoise, et a des impacts inévitables sur sa pédagogie.

\section{LA PÉDAGOGIE DE LA LITTÉRATURE À L’ÉCOLE CHINOISE \\ Principe pédagogique}

La norme shanghaïenne affirme que l'enseignement littéraire

"se réalise non seulement en classe, à travers des activités scolaires programmées et ayant des objectifs précis, mais aussi en dehors des classes, à travers des activités pratiques. Il faut créer des liens étroits entre la littérature et d'autres disciplines, entre l'étude scolaire et les pratiques extrascolaires, de façon à établir une pédagogie ouverte et adaptée au temps. ${ }^{13}$ ”

13. La norme shanghaïenne ... pour l'école primaire et secondaire, op.cit., 2004, p. 27. 
Cette affirmation a consolidé le statut de l'enseignement scolaire comme voie principale dans le développement de la compétence littéraire des élèves, et en même temps indiqué d'autres possibilités pour améliorer la culture, perfectionner le goût, favoriser la réflexion. Elle vise en effet à combiner une pédagogie littéraire statique, basée sur l'analyse des textes et les exercices scolaires, avec une autre pédagogie plus dynamique, qui s'appuie sur la pratique et l'expérience des élèves.

Une pédagogie dynamique est un processus qui vise à éveiller l'initiative et l'autonomie des élèves. Elle fait en sorte que les élèves acquièrent une idée générale du texte et soient touchés par son contenu; elle les fait ensuite expérimenter, c'est-à-dire mobiliser leurs expériences personnelles, afin d'éveiller en eux une sorte de résonance ou des questions à l'égard du texte ; enfin, elle cherche à faire comprendre aux élèves certaines règles de connaissances ou certains enseignements moraux à partir de leurs sentiments vis-à-vis du texte ; elle propose aux élèves de raisonner, de distinguer le vrai du faux, le bien du mal, le beau du laid - ce qui relève de l'appréciation littéraire.

Afin que les élèves puissent pratiquer et expérimenter de façon dynamique, la pédagogie chinoise de la littérature cherche à leur accorder suffisamment de temps et de liberté, les oriente et les guide dans la bonne direction, les aide à former l'esprit de doute et de recherche, et les encourage à former des opinions de façon autonome. Pour ce faire, l'école a prévu, dans l'enseignement scolaire de la littérature, un volet de "travaux pratiques" au sein des "cours élémentaires » et a créé, parallèlement, des "cours d'extension " au lycée. Elle a aussi formulé des propositions précises et concrètes pour les activités extrascolaires.

\section{Les aspects principaux de l'enseignement de la littérature}

\section{La lecture}

La lecture est considérée par les textes officiels de l'enseignement comme un acte personnel de l'élève. Ainsi, la norme nationale affirme que «la lecture collective doit veiller au sentiment, à l'expérience et à la compréhension particuliers de l'élève ", et que "l'enseignant ne doit pas remplacer la pratique de lecture de l'élève par sa propre analyse, ni l'expérimentation et la réflexion de l'élève par son explication ». Quant à l'élève, "il doit apprendre à résoudre les problèmes rencontrés dans la lecture à travers le travail en équipe, mais aussi se garder de laisser la discussion collective remplacer la lecture personnelle. ${ }^{14}$ »

14. La norme nationale ... pour l'enseignement obligatoire, op.cit., p. 22. 
Afin d'atténuer le caractère collectif de la lecture en classe et de favoriser la lecture personnelle et autonome, La norme nationale et La norme shanghaïenne ont non seulement défini le contenu et les exigences de la lecture collective en fonction des différentes étapes de la scolarité15, mais surtout la quantité minimale de lecture à effectuer durant chaque étape de la scolarité et la durée minimum de temps à consacrer chaque jour à la lecture par chaque élève selon son niveau ${ }^{16}$. Pour en garantir la mise en ouvre, elles ont prévu au moins trois mesures:

- instaurer spécialement des cours de « lecture personnelle » dans le programme scolaire, de façon à pousser les élèves dans la bibliothèque ou la salle de lecture ;

- demander aux rédacteurs des manuels de proposer aux élèves des listes d'ouvrages à lire pour chaque semestre, chaque titre d'ouvrage étant accompagné d'un résumé et d'un ou de plusieurs extraits ;

- l'enseignant a aussi l'obligation de recommander des ouvrages à lire en tenant compte de son enseignement, et d'organiser des échanges sur les ouvrages lus.

Sont également recommandées les lectures personnelles de la presse, dans une perspective de maîtrise de l'information, et celle des ouvrages littéraires canoniques. À l'heure actuelle, de nombreux élèves, au lieu de lire et d'aimer les ouvrages canoniques, préfèrent des ouvrages «fast food" ou "issus du Net». Selon certains experts, ce problème résulte, dans une certaine mesure, du manque de conseils des enseignants en matière de lecture personnelle des élèves. C'est pour cela que l'on insiste beaucoup en Chine sur le rôle de l'enseignant ainsi que sur l'influence de sa personnalité, afin de faire éprouver aux élèves le plaisir de la lecture des ouvrages canoniques, et de les imprégner de ces ouvrages.

L'objectif de la personnalisation de la lecture est d'une part d'aider l'élève à comprendre, à analyser, à synthétiser, à apprécier et à évaluer à partir de ses propres sentiments et expériences, et de l'autre de favoriser chez lui l'acquisition de bonnes habitudes de lecture, d'une méthode d'étude efficace, ainsi que l'adoption de valeurs correctes.

\section{Les travaux pratiques}

Les travaux pratiques sont classés pour l'essentiel en deux catégories: activité pratique et étude thématique. L'activité pratique, ayant des formes très diverses (interviews, enquêtes, débats, discours, rédaction des rapports ou des reportages, activités des sociétés littéraires, échanges ou études sur Internet, etc.), cherche à créer des situations concrètes de pratique pour l'élève et à orienter

15. Pour plus de détails, voir La norme nationale... pour l'enseignement obligatoire, op.cit., p. 8-17 et La norme shanghaïenne... pour l'école primaire et secondaire, op.cit., p. 32-34, p. 40-41.

16. Voir note 14 . 
son attention vers la société et la vie, de façon à développer sa conscience de participation, sa capacité à étudier et à résoudre des problèmes. Quant à l'étude thématique, elle vise à apprendre à l'élève à définir le thème à étudier selon son goût et sa préférence, à se documenter et à analyser, afin d'arriver finalement à produire un rapport de lecture ou un mémoire de recherche. Cette étude a pour objectif d'améliorer le niveau de raisonnement de l'élève et sa compétence d'appréciation et d'expression à l'aide de sa culture littéraire. Compte tenu de leur caractère ouvert, les travaux pratiques insistent notamment sur l'esprit autonome et le travail en équipe de l'élève, et accordent une importance particulière à l'utilisation des nouvelles technologies informatiques, ainsi qu'à la combinaison de la littérature avec d'autres formes d'art et de sciences.

\section{Les " cours d'extension"}

Les «cours d'extension», qui existent uniquement au lycée, sont optionnels. Beaucoup plus littéraires que les " cours élémentaires ", ils couvrent deux domaines : appréciation littéraire (poésie ancienne et moderne chinoise, roman et théâtre, essai, littérature biographique, textes scientifiques, etc.) et application (création de romans, de nouvelles et d'essais, rédaction de rapports, de reportages et du mémoires de recherche, maîtrise approfondie et application de connaissances lexicales, syntaxiques, rhétoriques et logiques dans la production écrite, etc. $).{ }^{17}$

Les cours d'extension sont considérés comme un approfondissement de l'enseignement de la littérature à l'école. Avec les cours élémentaires, ils constituent un système complet d'enseignement de la littérature à l'école chinoise.

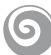

Bien que la Chine ait une longue tradition littéraire, la littérature n'a obtenu que très tardivement droit de cité à l'école en tant que partie constitutive du yuwen et discipline à part entière. À propos de son objectif, de son statut, de sa pédagogie, les débats n'ont jamais cessé et s'avèrent parfois vifs, mais au bout d'un siècle de pratique et d'exploration, grâce à des expériences réussies et des leçons tirées des échecs, les pédagogues et les enseignants chinois sont arrivés peu à peu à des consensus sur une pédagogie de la littérature conforme à la nature de l'enseignement et plus ou moins adaptée aux caractéristiques des élèves chinois ainsi qu'à la réalité de la Chine. Il est intéressant de montrer cette dimension de l'enseignement et de l'éducation de la Chine au monde, à une époque de mondialisation et de crise des modèles humanistes traditionnels.

17. Voir La norme shanghaïenne... pour l'école primaire et secondaire, op.cit. 2004, p. 43-44. 


\section{BIBLIOGRAPHIE}

Commission de l'éducation de la Municipalité de Shanghaï, La norme shanghaïenne $d u$ cours de yuwen pour l'école primaire et secondaire (Version d'essai), Éditions de l'éducation de Shanghai, 2004 (上海市教育委员会, “上海市中小学语文课程标准 》 (试行稿), 上海教育出版社, 2004年).

GU Zhichuan, "Réforme du programme de l'enseignement pré-universitaire et l'enseignement de yuwen à l'école secondaire", in Actes du $4^{e}$ colloque international sur les manuels et la pédagogie du chinois en Chine, People Education Press, le 13 avril 2006 (顾之川, 《基础教育课程改革与中学语文教学 》, in 《第四届中国语文课程教材教法国际研讨会论文》, 人民教育网，2006-04-13) http://www,pep,com,cn/gp/01_2/wb/kcjx/201205/t20120526_1126338,htm.

Ministère de l'éducation nationale de la République populaire de Chine, La norme nationale du cours de yuwen pour l'enseignement obligatoire, Presses de l'Université normale de Beijing, 2011 (中华人民共和国教育部, 《义务教育语文课程标准 》, 北京师范大学出版社, 2011年).

TAO Benyi (dir.), Manuel de Yuwen (de la $1^{\text {re }}$ à la $9^{\text {e }}$ années), Maison d'édition de l'Éducation de Shanghai (陶本一, 九年义务教学课本 《语文》, 上海教育出版社).

WANG Tiexian (dir.), Manuel de Yuwen (de la $10^{\mathrm{e}}$ à la $12^{\mathrm{e}}$ années), Presses de l'Université normale de la Chine de l'Est (王铁仙, 高级中学课本 《语文 》, 华东师范大学出版社). 